Full-length article

\title{
Recombinant adeno-associated virus-mediated human kallikrein gene therapy prevents high-salt diet-induced hypertension without effect on basal blood pressure ${ }^{1}$
}

\author{
Jiang-tao YAN ${ }^{2,4}$, Tao WANG ${ }^{2,4}$, Juan $\mathrm{LI}^{3}$, Xiao XIAO ${ }^{2,3}$, Dao-wen WANG ${ }^{2,5}$ \\ ${ }^{2}$ Department of Internal Medicine and Gene Therapy Center, Tongji Medical College of Huazhong University of Science and Technology, \\ Wuhan 430030, China; ${ }^{3}$ Molecular Pharmaceutics, The University of North Carolina School of Pharmacy, Chapel Hill, North Carolina, USA
}

\section{Key words}

recombinant adeno-associated virus vector; human tissue kallikrein; high-salt diet; hypertension

${ }^{1}$ This study was supported by grants from National Natural Science Foundation Committee (No 30571841$)$, National 863 project (No 2006 AA02A406), 973 project $(\mathrm{No}$ 2007CB512004).

${ }^{4}$ Jiang-tao YAN and Tao WANG contributed equally to this work.

${ }^{5}$ Correspondence to Prof Dao-wen WANG. Phn/Fax 86-27-8366-2842.

E-mail dwwang@tjh.tjmu.edu.cn

Received 2008-02-07

Accepted 2008-04-28

doi: $10.1111 / \mathrm{j} .1745-7254.2008 .00815 . \mathrm{x}$

\begin{abstract}
Aim: To investigate the effects of the expression of human kallikrein (HK) on basal level blood pressure and high-salt diet-induced hypertension. Methods: We delivered the recombinant adeno-associated viral (rAAV)-mediated HK (rAAVHK) gene and rAAV-LacZ (as the control) to normal, adult Sprague-Dawley rats. The animals were administered a normal diet in the first 4 weeks, followed by a high-salt diet. The expression of HK in the rats was assessed by ELISA and RTPCR. Blood pressure and $\mathrm{Na}^{+}$and $\mathrm{K}^{+}$urinary excretion were monitored. Results: Under the normal diet, no obvious changes in blood pressure and $\mathrm{Na}^{+}$and $\mathrm{K}^{+}$ urinary excretion were observed. When the high-salt diet was administered, systolic blood pressure in the control animals receiving rAAV-LacZ increased from $122.3 \pm 1.13 \mathrm{mmHg}$ to a stable $142.4 \pm 1.77 \mathrm{mmHg} 8$ weeks after the high-salt diet. In contrast, there was no significant increase in the blood pressure in the rAAV-HKtreated group, in which the blood pressure remained at $121.9 \pm 1.73 \mathrm{mmHg}$. In the rAAV-HK-treated group, $\mathrm{Na}^{+}$and $\mathrm{K}^{+}$urinary excretion were higher compared to those of the control group. The morphological analysis showed that HK delivery remarkably protected against renal damage induced by a high-salt intake. Conclusion: Our study indicates that rAAV-mediated human tissue kallikrein gene delivery is a potentially safe method for the long-term treatment of hypertension. More importantly, it could be applied in the salt-sensitive population to prevent the occurrence of hypertension.
\end{abstract}

\section{Introduction}

Hypertension is a chronic disease, with a high rate of morbility and low rate of treatment and control, which affects approximately 1 billion individuals worldwide ${ }^{[1]}$. Hypertension is the major risk factor of cardiovascular diseases, such as coronary heart diseases, heart failure, stroke, and kidney diseases. Clinical trails have demonstrated that antihypertensive therapy has been associated with 35\%-40\% reduction in stroke incidence, $20 \%-25 \%$ in infarction, and more than $50 \%$ in heart failure ${ }^{[2]}$.

The low control rate of hypertension is partly attributed to the current drugs, which are expensive and short lasting.
Gene therapy may provide an effective and stable control of blood pressure for hypertensive patients through long-termexpressing target genes ${ }^{[3]}$. The recombinant adeno-associated viral (rAAV) vector is an attractive vector for gene therapy protocols $^{[4-6]}$, which can mediate stable transduction and long-term expression of target genes in vivo, but without any known adverse effects. These features of rAAV make it possible that a single injection of the rAAV-mediated human kallikrein (rAAV-HK) target gene could control blood pressure of hypertensive patients.

The tissue kallikrein belongs to the serine proteinase family that cleaves a low molecular weight kininogen substrate 
to produce a vasodilator kinin peptide ${ }^{[7]}$. The binding of kinins to the bradykinin $\mathrm{B} 2$ receptor activates second messengers that trigger a broad spectrum of biological effects, such as vasodilation, smooth muscle contraction and relaxation, inflammation, pain, and cell proliferation ${ }^{[8]}$. The vasodilatory action of the kallikrein-kinin system (KKS) counterbalances the vasoconstrictive actions of the reninangiotensin system. Our previous studies have shown that the overexpression of HK mediated by rAAV decreases blood pressure and ameliorates the secondary injuries of organs associated with hypertension in spontaneous hypertensive rats in the long term ${ }^{[9]}$. However, the $\mathrm{AAAV}-\mathrm{HK}$ gene delivery lacks expression regulation. Therefore, whether the overexpression of the HK gene can cause hypotension, a dangerous condition for hypertensive patients, needs to be examined. To identify the effects of the rAAV-HK gene on normal blood pressure is critical and necessary before any potential clinical applications.

The positive relationship between sodium intake and blood pressure has been well established in ecological, epidemiological, and experimental human studies ${ }^{[10-14]}$. Renal kallikrein is synthesized in the connecting tubule cells in the distal nephron, and is involved in the homeostasis of sodium and water balance in the kidney. Its attenuation is suggested as a possible etiological factor in salt-sensitive hypertension ${ }^{[15,16]}$. Our previous studies have demonstrated that $\mathrm{rAAV}-\mathrm{HK}$ delivery attenuates the increased blood pressure induced by fructose or streptozotocin in conjunction with a high-fat diet and ameliorates organ injury ${ }^{[17]}$. However, its effect on high-salt diet-induced hypertension in rats with normal blood pressure remains unknown.

In the present study, we delivered the $\mathrm{AAV}-\mathrm{HK}$ gene to normal, adult Sprague-Dawley (SD) rats and determined the effects of overexpression of human tissue kallikrein on the basal level of blood pressure and high-salt diet-induced hypertension.

\section{Material and methods}

Construction plasmid ArAAV vector plasmid, pXXUF1, and rAAV plasmid containing a report gene, pdxII-LacZ as the control, packaging plasmid pXX2, and adenovirus helper plasmid pXX6, were described previously ${ }^{[18]}$. The pXXUF1 contains 2 inverse terminal repeats, cytomegalovirus promoter (CMV) and a poly A tail. A 860 base pair HK fragment (Not $\mathrm{I} /$ Not I ) containing an open reading frame was subcloned into pXXUF1 downstream of CMV to the resultant plasmid pUF1-HK.

Production and measurement of titers of $\mathrm{AAAV}$ vectors rAAV-HK and rAAV-LacZ were produced as described pre- viously ${ }^{[9]}$. Human 293 cells (adenovirus 5-sheared DNA transferred embryonic kidney epithelial cells) were grown in Dulbecco's modified Eagle's medium (DMEM; Gibco) supplemented with $10 \%$ fetal bovine serum (FBS; Gibco) and antibiotics in a $5 \% \mathrm{CO}_{2}$ atmosphere at $37^{\circ} \mathrm{C}$. Immediately before transfection, $15 \mathrm{~mL}$ fresh DMEM containing $10 \%$ FBS was added to each $15 \mathrm{~cm}$-diameter plate of the cells (70\%-80\% confluency). A total of $85 \mu \mathrm{g}$ plasmid DNA (ratio of pUF1.HK or pdxII-LacZ:pXX2:pXX6 was 1:1:1 in molar) was dissolved in $2.5 \mathrm{~mol} / \mathrm{L} \mathrm{CaCl}_{2}$ and then quickly mixed with $1 \times$ BES-buffered salineand added to the cells. After 8-12 h of transfection, the medium was replaced with fresh DMEM containing $10 \%$ FBS, and the cells were harvested at $48-72 \mathrm{~h}$ post-transfection. After low-speed centrifugation on a tabletop centrifuge, the cell pellets wereresuspended in 1-2 $\mathrm{mL}$ of $100 \mathrm{mmol} / \mathrm{L} \mathrm{NaCl}$ and $10 \mathrm{mmol} / \mathrm{L}$ Tris- $\mathrm{HCl}$ (pH 8.0) and subjected to 3 cycles of freeze-thaw; cell debris was removed by centrifuge. For the purification of rAAV, a single-step gravity-flow column purification method was carried out as described by Auricchio et $a l^{[19]}$. The eluted rAAV was aliquoted and stored at $-80^{\circ} \mathrm{C}$ for the experiments.

The titer of rAAV-LacZ and rAAV-HK was determined by dot blot hybridization.

Animal treatment and gene delivery A total 12 male SD rats (3 months old), weighing 250-280 g, were randomly divided into 2 groups ( $n=6$ each group). The rats were housed at room temperature with a $12 \mathrm{~h}$ light/dark cycle and fed normal rat chow and tap water. All animal experimental protocols were approved by the Institutional Animal Research Committee of Tongji Medical College (Wuhan, China), and were carried out according to the guidelines of the National Institutes of Health.

For gene delivery, the animals were anesthetized by an intraperitoneal injection of pentobarbital at a dose of $50 \mathrm{mg} / \mathrm{kg}$ body weight. The SD rats were administered a single tail vein injection of $\mathrm{rAAV}-\mathrm{HK}$ or $\mathrm{AAV}-\mathrm{LacZ}$ (approximately $1 \times 10^{11}$ transducing units in $1 \mathrm{~mL}$ saline solution), respectively. After the injection, the rats were kept warm by an infrared lamp until they regained consciousness.

At week 5 after the $\mathrm{AAAV}$ injection, the animals were fed a standard rodent diet containing $8 \% \mathrm{NaCl}$ for 8 weeks. All the animals were killed at 13 weeks after rAAV delivery under pentobarbital anesthesia ( $50 \mathrm{mg} / \mathrm{kg}$ body wt), and each rat's heart, lung, kidney, muscle, and liver were collected in liquid nitrogen and then stored at $-80{ }^{\circ} \mathrm{C}$ until further experiments were performed.

Systolic blood pressure measurement From the beginning of the experiment, the systolic blood pressure of the rats was measured weekly with a manometer-tachometer (Rat 
Tail NIBP System; ADI Instruments) by cuffing the tails of the animals. This device requires minimal warming of rats (usually $15 \mathrm{~min}$ ) before blood pressure determination and a brief period of restraint in a plastic cage. For each animal, the systolic blood pressure was represented as the mean of at least 5 recordings.

Collection of urine The urinary volume of each animal was measured weekly. The $24 \mathrm{~h}$ urine samples were collected in metabolic cages with $500 \mu \mathrm{L}$ toleuene to prevent the decay of urine and stored at $-80^{\circ} \mathrm{C}$ until used for the measurements of the kallikrein levels, urinary osmotic pressure, and urinary excretion of $\mathrm{Na}^{+}$and $\mathrm{K}^{+}$.

RT-PCR analysis of human tissue kallikrein mRNA An RT-PCR analysis specific for HK mRNA was carried out using the RT-PCR kit (TAKARA Biotechnology). Total RNA was extracted from rat tissues by TRIZol reagent (Gibco), and was used for the synthesis of cDNA with AMV reverse transcriptase. The resulting cDNA sample was PCR amplified with HK-specific primers (forward 5'-CCACCATGGGGTTCCTGGTT-3' and reverse 5'-CGCGGATCCACATTTGATT T-3') and the $\beta$-actin primer (forward, 5'-GGAGAAGATGACCCAGATC-3' and reverse, 5'-GATCTTCATGAGGTAGTCAG-3') as the control, respectively, under the following conditions: a denaturing phase of $1 \mathrm{~min}$ at $94^{\circ} \mathrm{C}$, annealing phase of $40 \mathrm{~s}$ at $65^{\circ} \mathrm{C}$, and extension phase of $1 \mathrm{~min}$ at $72{ }^{\circ} \mathrm{C}$ for 20 cycles.

ELISA specific for human tissue kallikrein in urine ELISA reagents specific for human tissue kallikrein were supplied by Dr Lee CHAO (Department of Biochemistry and Molecular Biology, Medical University of South Carolina, SC, USA). The ELISA analysis for human tissue kallikrein in the animal urine samples was performed according the method described previously ${ }^{[9]}$. The plates were read at $405 \mathrm{~nm}$ on an ELX 800 ELISA reader (Bio-Tek Instruments).

Analysis of morphology and collagen deposition A part of the kidney for each animal was preserved in $4 \%$ phosphate-buffered saline-buffered formaldehyde solution and embedded in paraffin. Four micrometer-thick sections were cut and stained with Sirius red (collagen stains red with Sirius red staining) using the method previously described ${ }^{[20]}$.

Statistical analysis The results were expressed as mean \pm SEM. The statistical significance of the difference in systolic blood pressure between SD rats receiving $\mathrm{rAAV}-$ LacZ and rAAV-HK was determined by ANOVA. In addition, we used an unpaired Student's $t$-test to assess the differences of the HK levels in urine, body weight, urinary volume, and urinary osmotic pressure between the rAAV-LacZ and rAAV-HK groups after gene delivery.

\section{Results}

Expression of human tissue kallikrein mRNA in SD rats The mRNA levels of human tissue kallikrein in the heart, renal, lung, liver, and muscle tissues in SD rats were examined by RT-PCR at 13 weeks after an intravenous injection of rAAV. The results showed that human tissue kallikrein mRNA was expressed in all tested tissues in rAAV-HKtreated rats, but not in $\mathrm{rAAV}-\mathrm{LacZ}$-treated rats (Figure 1).

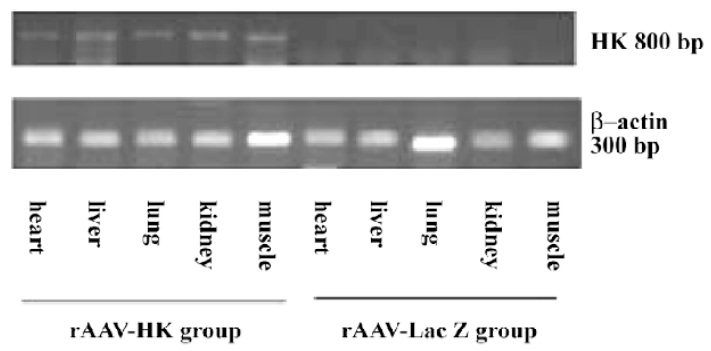

Figure 1. Transcription of human tissue kallikrein in SD rats injected intravenously with $\mathrm{rAAV}-\mathrm{HK}$ and $\mathrm{rAAV}-\mathrm{LacZ}$ was detected by RT-PCR. HK mRNA was detected in all of the main tissues in the rAAV-HK-treated rats, but not in the rAAV-LacZ-treated rats.

Immunoreactive human tissue kallikrein levels in the urine of SD rats The ELISA specific for human tissue kallikrein was used to detect the immunoreactive human tissue kallikrein levels in the urine of SD rats. No immunoreactive human tissue kallikrein was detected in the urine of rats with rAAV-LacZ delivery. The immunoreactive human tissue kallikrein levels in the urine in SD rats reached 3.67 $\pm 0.135 \mu \mathrm{g} / \mathrm{mL}$ at 2 weeks after rAAV-HK injection, and maintained at approximately $4.32 \pm 0.268 \mu \mathrm{g} / \mathrm{mL}$ until the end of the experiment. These results demonstrated that $\mathrm{AAV}-\mathrm{HK}$ transfer can drive the HK gene to express the target protein stably in vivo in the long term (Figure 2).

Blood pressure regulation effects of $\mathrm{PAAV}-\mathrm{HK}$ in SD rats Under the normal diet, no change in blood pressure was observed in all SD rats after the injection of rAAV-HK or rAAV-LacZ. When a high-salt diet was given, the systolic blood pressure of the rats receiving $\mathrm{rAAV}-\mathrm{LacZ}$ increased from $122.3 \pm 1.13 \mathrm{mmHg}$ to a stable $142.4 \pm 1.77 \mathrm{mmHg}$ in 8 weeks after being fed the high-salt diet. However, the blood pressure in the rAAV-HK-treated SD rats had no significant changes on the condition of different diet intake, which remained at approximately $121.9 \pm 1.73 \mathrm{mmHg}$. After the highsalt diet was given, the systolic blood pressure in rats receiving rAAV-HK was significantly lower than that in rAAVLacZ-injected rats $(P<0.05, n=6$, ANOVA), and the antihypertensive effect of $\mathrm{AAAV}-\mathrm{HK}$ lasted until the end of the experiment (Figure 3). 


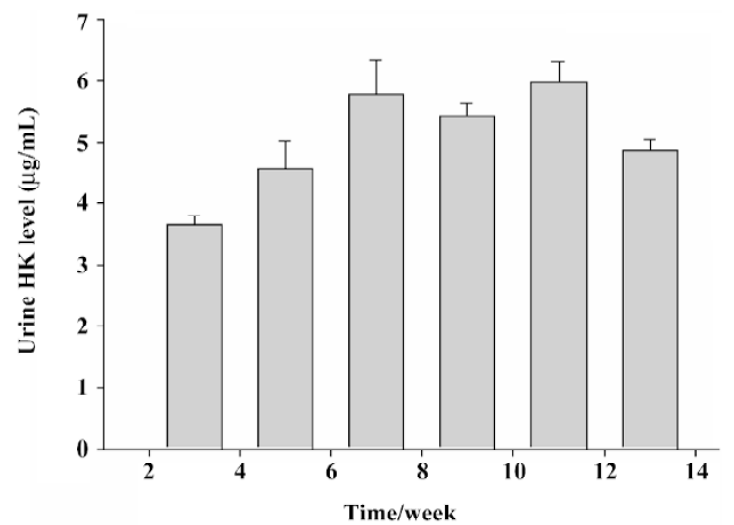

Figure 2. Time-course of human tissue kallikrein expression in SD rats. Urine was collected biweekly and human tissue kallikrein concentrations were measured by ELISA. Immunoreactive HK was detected in the urine from the second week after injection, and the urinary $\mathrm{HK}$ level was at $4.32 \pm 0.268 \mu \mathrm{g} / \mathrm{mL}$. No immunoreactive HK was detected in the urine of control rats receiving $\mathrm{rAAV}-\mathrm{LacZ}$ (data not shown).

Physiological parametersin SD rats injected with rAAVHK or rAAV-LacZ Under the normal diet, no changes in urinary volume, urinary osmotic pressure, and urinary excretion of $\mathrm{Na}^{+}$and $\mathrm{K}^{+}$were observed in all SD rats after $\mathrm{rAAV}$ delivery. When the high-salt diet was given, urine volume, urinary osmotic pressure, and urinary excretion of $\mathrm{Na}^{+}$and $\mathrm{K}^{+}$increased in the $\mathrm{rAAV}-\mathrm{HK}$-treated animals significantly more than the rAAV-LacZ-treated animals (Table 1).

Protective effect of $\mathrm{AAAV}-\mathrm{HK}$ injection on the kidney Sirus red staining was used to analyze the collagen deposition in the kidney of rats given a high-salt diet for 8 weeks. As shown in Figure 4, there was much more intensive collagen deposition in the glomerulus in the control group than that in the rAAV-HK-treated animals. Sirus red-stained sections were further analyzed by using HAIPS Pathologic Imagic Analysis System for the percentage of extracellular matrix (ECM)/collagen area surface. ECM was markedly reduced in the $\mathrm{AAV}-\mathrm{HK}$-treated animals compared with the rAAV-LacZ-treated control group $(19.36 \% \pm 5.11 \% \mathrm{vs}$

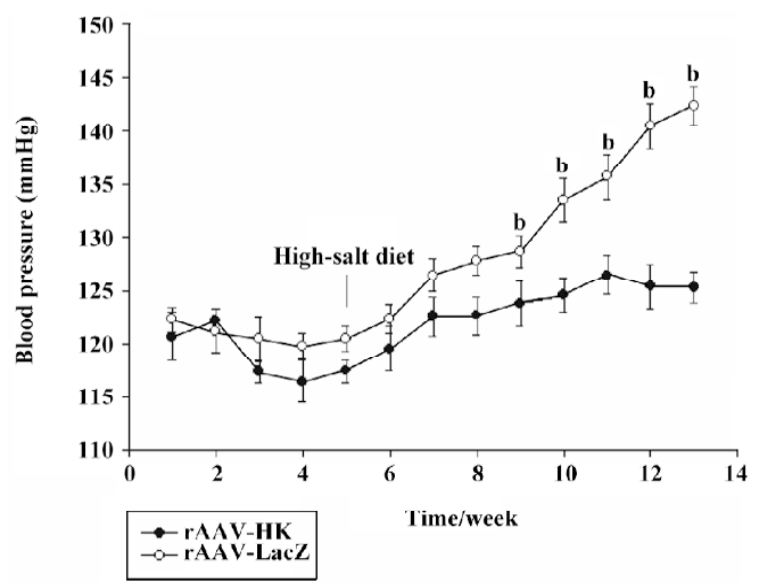

Figure 3. Regulation of blood pressure in SD rats injected intravenously with rAAV-HK and rAAV-LacZ. Under the normal diet, no change in the blood pressure was observed. When the high-salt diet was given, the systolic blood pressure in the control animals receiving rAAV-LacZ increased from $122.3 \pm 1.13 \mathrm{mmHg}$ to $142.4 \pm 1.77$ $\mathrm{mmHg}$ in 8 weeks. In contrast, the blood pressure in the rAAV-HKtreated group had no obvious increase (approximately 121.9 \pm 1.73 $\mathrm{mmHg}$ ), and was lower significantly than that of the rAAV-LacZtreated group ( ${ }^{\mathrm{b}} P<0.05, n=6$, ANOVA). The antihypertensive effect of rAAV-HK lasted until the end of the experiment.

$3.36 \% \pm 1.97 \%, n=5, P<0.05)$. These findings indicated that HK gene treatment significantly prevented collagen deposition in the kidney of high salt-induced hypertensive rats (Figure 4).

\section{Discussion}

Our study demonstrated that a single intravenous injection of rAAV-HK could prevent high-salt diet-induced hypertension in SD rats. It also showed that the antihypertensive effect lasted throughout the duration of the experiment without any effect on the basal blood pressure in SD rats fed a normal diet. Furthermore, human tissue kallikrein delivery significantly increased the urinary osmotic pressure and urinary excretion of $\mathrm{Na}^{+}$and $\mathrm{K}^{+}$, as well as reduced collagen deposition in the kidney in rats fed a high-salt diet. These

Table 1. Physiological parameters in SD rats injected with rAAV-HK and rAAV-LacZ and fed a normal diet or high-salt diet. Data are mean \pm SEM. $n=6 .{ }^{\mathrm{b}} P<0.05$ compared to rAAV-LacZ group, $t$-test.

\begin{tabular}{|c|c|c|c|c|}
\hline & \multicolumn{2}{|c|}{ rAAV-LacZ group } & \multicolumn{2}{|c|}{ rAAV-HK group } \\
\hline & Normal diet & High-salt diet & Normal diet & High-salt diet \\
\hline Urine volume (mL/day) & $2.3 \pm 0.11$ & $15.6 \pm 1.12$ & $2.4 \pm 0.23$ & $21.8 \pm 2.13^{\mathrm{b}}$ \\
\hline Urinary osmotic pressure $\left(\mathrm{mOsm} / \mathrm{kgH}_{2} \mathrm{O}\right)$ & $897 \pm 22.5$ & $913 \pm 31.9$ & $779 \pm 23.5$ & $1178 \pm 35.9^{\mathrm{b}}$ \\
\hline Urine $\mathrm{Na}^{+}(\mathrm{mmol} / \mathrm{day})$ & $2.26 \pm 0.128$ & $18.9 \pm 2.43$ & $2.37 \pm 0.229$ & $25.6 \pm 2.75^{\mathrm{b}}$ \\
\hline Urine $\mathrm{K}^{+}$(mmol/day) & $1.36 \pm 0.135$ & $2.06 \pm 0.211$ & $1.42 \pm 0.147$ & $2.65 \pm 0.183^{\mathrm{b}}$ \\
\hline
\end{tabular}



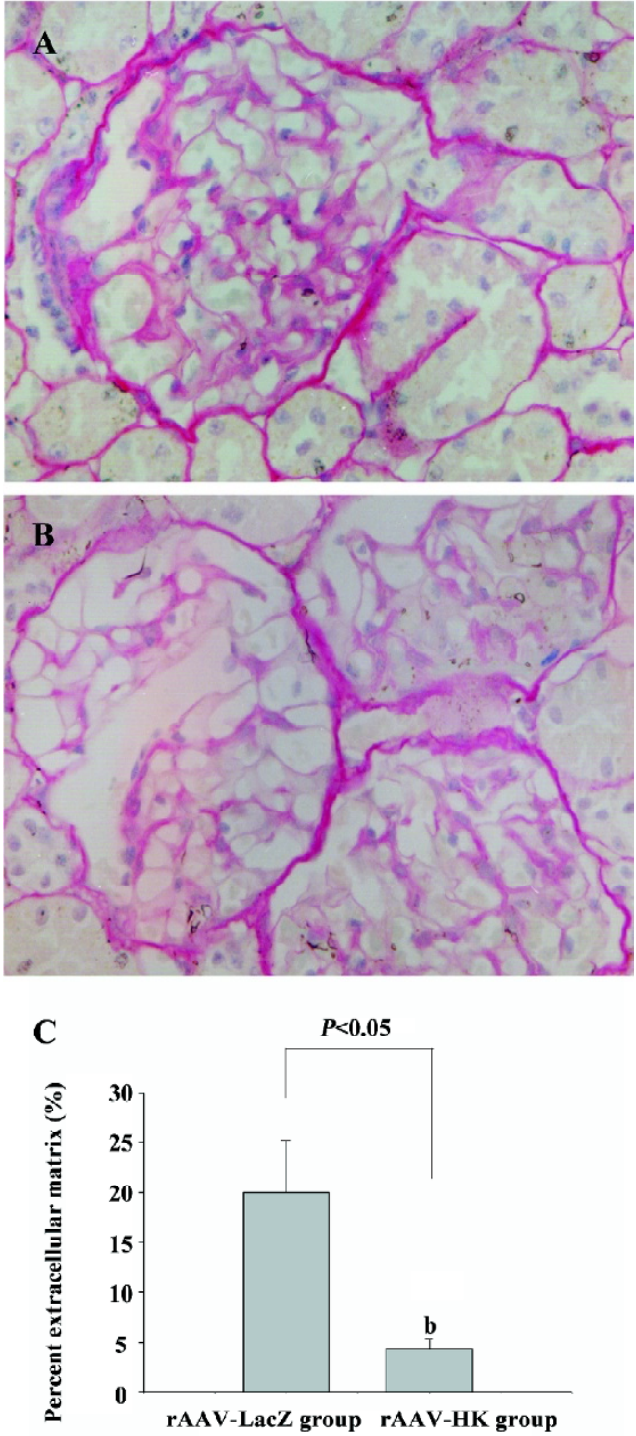

Figure 4. Fibrosis and collagen deposition in SD rat kidneys fed a high-salt diet. Sirus red staining showed that in the untreated control SD rats (A) Sirus staining (representing the collagen deposition) was more intense in the glomerulus than the rAAV-HK-treated SD rats (B). Sirus red-stained sections were analyzed by using HAIPS Pathologic Imagic Analysis System for the percentage of ECM/collagen area surface. ECM was markedly reduced in $\mathrm{rAAV}-\mathrm{HK}$-treated animals compared with the rAAV-LacZ-treated control group $(19.36 \% \pm 5.11 \% v s$ $\left.3.36 \% \pm 1.97 \%, n=5,{ }^{\mathrm{b}} P<0.05\right)(\mathrm{C})$.

findings indicated that $\mathrm{rAAV}-\mathrm{HK}$ delivery resulted in a longterm and stable expression of human tissue kallikrein and had a significant protective effect against high-salt diet-induced hypertension and kidney injury in SD rats. In addition, rAAV-HK delivery did not lower the normal blood pressure on rats fed a normal diet, which suggested that rAAV-HK gene therapy might be the ideal treatment for hypertension.

In the gene therapy research area of hypertension, there are many problems that need to be resolved, such as, how to control the reduction extent of blood pressure and potential risk of over-reduction of blood pressure in hypertensive patients. Therefore, the choice of vectors for gene delivery and the selection of the targeting gene are critical for the successful gene therapy strategy. The rAAV vector is an attractive vector for gene therapy protocols. It has the ability to remain expressed throughout the full life cycle of a cell by integrating into host cellular DNA or remaining episomal inside the cell. This unique feature can render a long-term and stable expression of the target gene ${ }^{[4-6]}$. Previous studies have shown that a single injection of $\mathrm{rAAV}-\mathrm{HK}$ gene delivery causes a prolonged reduction of systolic blood pressure in different hypertensive animal models, which makes it possible to treat hypertension through gene therapy ${ }^{[9,17]}$. However, it remains unknown whether the overexpression of the HK gene can induce hypotension in normotensive rats. Our study demonstrated that the expression of human tissue kallikrein delivered by rAAV would not cause hypotension in SD rats with normotensive SD rats. Endogenous bradykinin is suggested to be involved in the vasodilation or the hypotensive effects of ACE inhibitors using shortacting peptide B2 receptor antagonists. However, endogenous bradykinin is found not to contribute to the regulation of blood pressure under basal conditions ${ }^{[21-24]}$, which is consistent with our results. Our data are further supported by a recent study of the bradykinin $\mathrm{B} 2$ receptor in null mice. In these mice, the lack of B2 receptor from early development did not alter the maturation of blood pressure under conditions of normal sodium intake ${ }^{[25]}$.

Interestingly, our results showed that human tissue kallikrein gene delivery in normal SD rats had no effect on the excretion of natrium from the kidney under normal sodium intake, although it has been known that renal KKS is involved in the excretion of natrium in the kidney ${ }^{[15]}$. The renal KKS is composed of renal kallikrein, its inhibitor (kallistatin), low molecular weight kininogen, 2 tubular-specific kinases, and kinin receptors. All components of the renal KKS are localized along the renal distal tubules, particularly from the connecting tubules to collecting ducts. However, it is also well known that $90 \%-95 \%$ of sodium is reabsorbed in the proximal portion and particularly in the loop of Henle before it reaches the distal nephron in the kidney, which could explain that human tissue kallikrein gene delivery does not increase the excretion of natrium from the kidney with normal sodium intake.

In our study, we found that a high-salt diet induces hypertension in normal SD rats; however, rAAV-human tissue kallikrein delivery could protect against the increase of blood 
pressure with a high-salt diet. Research results from a normotensive population demonstrate that sodium loading induces a change of blood pressure that is associated with the basal urinary excretion of renal kallikrein, although sodium loading decreases the urinary excretion of renal kallikrein ${ }^{[26]}$. Human tissue kallikrein gene overexpression decreases the sensitivity to a high-salt diet. Our study indicated that the SD rats with human tissue kallikrein gene delivery had a higher excretion of urinary natrium compared to the control rats fed a high-salt diet. These data suggested that rAAVmediated kallikrein delivery counterparts the high-salt dietinduced renal kallikrein decrease, increases the urinary natrium excretion, and consequently protects against blood pressure increase.

The renal KKS may be involved in the renal vasodilation during high natrium intake, and hence a functionally normal KKS is important to prevent salt-sensitive hypertension and secondary renal injuries. As shown in our study, human tissue kallikrein gene treatment significantly prevented the collagen deposition in the kidney of high-salt diet-induced hypertensive rats.

In summary, we report that $\mathrm{AAAV}$-mediated human tissue kallikrein gene delivery does not decrease the basal blood pressure in normotensive rats fed a normal diet. However, it significantly protects against hypertension induced by a high-salt diet and secondary renal injuries. Our study suggested that rAAV-mediated human tissue kallikrein gene delivery is a safe way to treat hypertension, and could have potential when applied to the salt-sensitive population to prevent the occurrence of hypertension.

\section{Author contribution}

Dao-wen WANG designed research; Jiang-tao YAN, Tao WANG performed research; Juan LI, Xiao XIAO contributed analytical reagents and tools; Jiang-tao YAN, Tao WANG analyzed data; Jiang-tao YAN wrote the paper.

\section{References}

1 Chobanian AV, Bakris GL, Black HR, Cushman WC, Green LA, Izzo JL Jr, et al. The seventh report of the joint national committee on prevention, detection, evaluation and treatment of high blood pressure: The JNC 7 report. JAMA 2003; 289: 256072 .

2 Neal B, Macmahon S, Chapman N. Effects of ACE inhibitors, calcium antagnosists, and other blood-pressure-lowering drugs. Lancet 2000; 356: 1955-64.

3 Phillips MI. Gene therapy for hypertension: the preclinical data. Hypertension 2001; 38 (Part 2): 543-8.

4 Xiao X, Li J, Samulski RJ. Production of high-titer recombinant adenoassociated virus vectors in the absence of helper virus. $\mathrm{J}$
Virol 1998; 72: 2224-32.

5 Sun L, Li J, Xiao X. Overcoming adeno-associated virus vector size limitation through viral DNA heterodimerization. Nat Med 2000; 6: 599-602.

6 Monahan PE, Samulski RJ. AAV: is clinical success in the horizon? Gene Therapy 2000; 7: 24-30.

7 Bhoola KD, Figueroa CD, Worthy K. Bioregulation of kinins: kallikreins, kininogens, kininases. Pharmacol Rev 1992; 44: 18 .

8 Chao J, Chao L. Kallikrein-kinin in stroke, cardiovascular and renal diseases. Exp Physiol 2005; 90: 291-8.

9 Wang T, Li H, Zhao C, Chen C, Li J, Chao J, et al. Recombinant adeno-associated virus-mediated kallikrein gene therapy reduces hypertension and attenuates its cardiovascular injuries. Gene Ther 2004; 11: 1342-50.

10 Weinberger M. Salt sensitivity of blood pressure in humans. Hypertension 1996; 27: 481-90.

11 Weinberger M. Salt sensitivity: does it play an important role in the pathogenesis and treatment of hypertension? Curr Opin Nephrol Hypertens 1996; 5: 205-8.

12 Weinberger M. Salt and blood pressure. Curr Opin Cardiol 2000; 15: 254-7.

13 Weinberger M. More on the sodium saga. Hypertension 2004; 44: 609-11.

14 Weinberger M. Sodium and blood pressure 2003. Curr Opin Cardiol 2004; 19: 353-6.

15 Katori M, Majima M. A missing link between a high salt intake and blood pressure increase. J Pharmacol Sci 2006; 100: 37090

16 Katori M, Majima M. The renal kallikrein-kinin system: its role as a safety valve for excess sodium intake, and its attenuation as a possible etiologic factor in salt-sensitive hypertension. Crit Review Clin Lab Sci 2003; 40: 43-115.

17 Yuan G, Deng J, Wang T, Zhao C, Xu X, Wang P, et al. Tissue kallikrein reverses insulin resistance and attenuates nephropathy in diabetic rats by activation of phosphatidylinositol 3-kinase/ protein kinase B and adenosine 5'-monophosphate-activated protein kinase signaling pathways. Endocrinology 2007; 148: 2016-26.

18 Xiao X, Li J, Samulski RJ. Production of high-titer recombinant adenoassociated virus vectors in the absence of helper adenovirus. J Virol 1998; 72: 2224-32.

19 Auricchio A, Hildinger M, Connor E, Gao GP, Wilson JM. Isolation of highly infectious and pure adeno-associated virus type 2 vectors with a singlestep gravity-flow column. Hum Gene Ther 2001; 12: 71-6.

20 Wang C, Dobrzynski E, Chao J, Chao L. Adrenomedullin gene delivery attenuates renal damage and cardiac hypertrophy in Goldblatt hypertensive rats. Am J Physiol Renal Physiol 2001; 280: F964-F971

21 Gainer JV, Morrow JD, Loveland A, King DJ, Brown NJ. Effect of bradykinin-receptor blockade on the response to angiotensinconverting-enzyme inhibitor in normotensive and hypertensive subjects. N Engl J Med 1998; 339: 1285-92.

22 Hornig B, Kohler C, Drexler H. Role of bradykinin in mediating vascular effects of angiotensin-converting enzyme inhibitors in humans. Circulation 1997; 95: 1115-8.

23 Squire IB, O'Kane KP, Anderson N, Reid JL. Bradykinin B(2) 
receptor antagonism attenuates blood pressure response to acute angiotensin-converting enzyme inhibition in normal men. Hypertension 2000; 36: 132-6.

24 El-Dahr SS, Dipp S, Meleg-Smith S, Pinna-Parpaglia P, Madeddu P. Fetal ontogeny and role of metanephric bradykinin B2 receptors. Pediatr Nephrol 2000; 14: 288-96
25 Alfie ME, Sigmon DH, Pomposiello SI, Carretero OA. Effect of high salt intake in mutant mice lacking bradykinin-B2 receptors. Hypertension 1997; 29: 483-7.

26 Bönner T, Thieven B, Rütten H, Chrosch R, Krone W. Renal kallikrein is a determinant of salt sensitivity. J Hypertens 1993; 11 Suppl 5: S210-211.

\title{
2008 International Conference on Ginseng
}

\author{
Changchun, China
}

Sep 1-3, 2008

Please contact with Dr Ji CHEN.

Phn 10-6316-5182

E-mailchenj@imm.ac.cn; ginseng2008@126.com 